- Galloy restorations in moderate to large class I and class II cavities displayed early catastrophic failure that resulted in fractured tooth structure in 60\% of cases.

- Galloy restorations demonstrate dimensional instability and marked corrosion in the presence or oral fluids even when placed using procedures designed to reduce moisture contamination.

- Due to its inferior clinical performance, Galloy is unsuitable as a restorative material for use in moderate to large class I and class II restorations.

\title{
A 3-year longitudinal, controlled clinical study of a gallium-based restorative material
}

\author{
S. M. Dunne, ${ }^{1}$ R. Abraham ${ }^{2}$ and C. L. Pankhurst ${ }^{3}$
}

\begin{abstract}
Aim The aim of this three-year longitudinal controlled study was to compare the clinical performance of Galloy ${ }^{1}$ versus a high copper, mercury based Dispersalloy ${ }^{2}$ filling material.

Methods Moderate to large class I and class II cavities or replacement restorations were selected and 25 Galloy ${ }^{\circledR}$ restorations and 25 Dispersalloy controls were placed in 14 adult patients by a single operator. Restorations were photographed and a silicone impression recorded at baseline, 6 months, 1 year, 2 years and 3 years.

Results At 3 years all 22 Dispersalloy restorations but only 4 Galloy restorations were still in situ. Three Dispersalloy restorations were lost to follow-up. Tooth fracture was observed in 15 (60\%) of the Galloy restorations by the end of the 3 years, compared to one (4\%) Dispersalloy restoration, which failed due to tooth fracture. A further six Galloy restorations had to be removed due to complaints of persistent pain. Four teeth restored with Galloy required endodontic treatment but none of the Dispersalloy restored teeth required endodontics. Of the four Galloy restorations remaining in situ, three were relatively small restorations and the fourth a moderate sized restoration required a marginal repair.

Conclusion The clinical performance of Galloy restorations was so grossly inferior to the Dispersalloy controls that Galloy cannot be recommended for clinical use in moderate to large or multi-surface cavities.
\end{abstract}

\section{INTRODUCTION}

The substitution of a gallium alloy for mercury in amalgam was suggested by Puttkamer as long ago as $1928 .{ }^{1}$ Gallium, like mercury, has a low melting point and at room temperature reacts with other metals to form a workable material that hardens over time. Despite the recognition of these advantageous characteristics, a Galloy substituted restorative material was not produced until 1956. ${ }^{2}$ The first gallium alloy for dental purposes ${ }^{2}$ alloyed liquid gallium with powders of Ta (Tantalum), Cr (Chromium), Mo (Molybdenum), In (Indium), Co (Cobalt), Nickel (Ni), Gold

\footnotetext{
${ }^{1 *}$ Professor, ${ }^{2}$ Senior Specialist Clinical Tutor, Department of Primary Dental Care and ${ }^{3}$ Lecturer, Unit of Distance Education, Guy's, King's, St Thomas (GKT) Dental Institute, King's College London,

*Correspondence to: Professor S M Dunne, Department of Primary Dental Care, GKT London Dental Institute, Denmark Hill Campus, Caldecot Road, London SE5 9RW United Kingdom Email:s.dunne@kcl.ac.uk
}

\section{Refereed paper}

Received 02.04.03; Accepted 24.03.04

doi: $10.1038 /$ sj.bdj.4812175

๑ British Dental Journal 2005; 198: 355-359
(Au) and some $\mathrm{Cu}$ (Copper)-Sn (Tin) alloys. The $\mathrm{Cu}-\mathrm{Sn}$ alloys produced mixes that hardened satisfactorily. ${ }^{3}$ Waterstrat ${ }^{4}$ formulated Ga (Gallium)-Pd (Palladium)-Sn (Tin) alloys and found their strength and setting expansion to be acceptable. Interest in the development of gallium alloys waned until the 1990s. Media and public concern about possible toxicity and environmental pollution associated with mercury containing silver amalgam reawakened the pursuit of mercury-free alternatives ${ }^{5,6}$ and drove the technical development of new gallium alloys.

Two distinct types of preparations of Gallium containing alloys became available for clinical use, those containing palladium at 9\% (Gallium Alloy GF, Tokurike Honten, Tokyo, Japan) or 2\% Gallium GFII and palladium-free alloys namely Galloy ${ }^{\circledR}$ (Southern Dental Industries, Bayswater, Australia). Unfortunately, early studies with the 9\% palladium alloy indicated poor clinical performance with marked discolouration, surface roughness and marginal breakdown. The performance of the reformulated Gallium GFII in laboratory studies was superior, exhibiting fewer defects associated with corrosion, but the setting expansion was much greater than that exhibited by silver amalgam. ${ }^{7}$

Galloy ${ }^{\circledR}$ (a non-palladium gallium alloy containing a spherical high copper alloy powder Ag (Silver) (60.1\%), Sn (28.05\%), Cu $(11.8 \%)$ and PT (Platinum) $(0.05 \%)$ ) and a Ga alloy liquid (Ga (62\%), In (Indium) (25\%), Sn (13\%) and Bi ( Bismuth ) (0.05\%) ${ }^{7}$ was introduced in Australia in 1994. The alloying of Ga with In and Sn brings the melting point down from $29.8^{\circ} \mathrm{C}$ for pure $\mathrm{Ga}$ to about $10^{\circ} \mathrm{C}$, rendering it suitable for dental purposes. The material was accompanied by manufacturers' claims for a lower setting expansion and improved clinical performance, as a result of changes in formulation and recommended encapsulation procedures. Gallium has the ability to wet materials including teeth and metals indicating a potential for improved adaption and marginal seal. ${ }^{8}$ However, wetablity also promotes unwanted adherence to dental instruments impairing manipulation. To enhance the ease of handling, Galloy ${ }^{\circledR}$ is delivered via a direct placement capsule system (see Figure 1). The material was approved by the United States Food and Drug Administration and the Scandinavian Institute of Dental Materials. Despite the manufacturers' recommendation that Galloy is indicated for use in conservative class I and class II posterior restorations, no restrictions on its use in moderate or larger cavities have been placed.

Initial laboratory studies of Galloy ${ }^{\circledR}$ supported its potential as an acceptable restorative material. Published short-term interim results from clinical trials conducted by Osborne and Summitt ${ }^{9,10}$ 


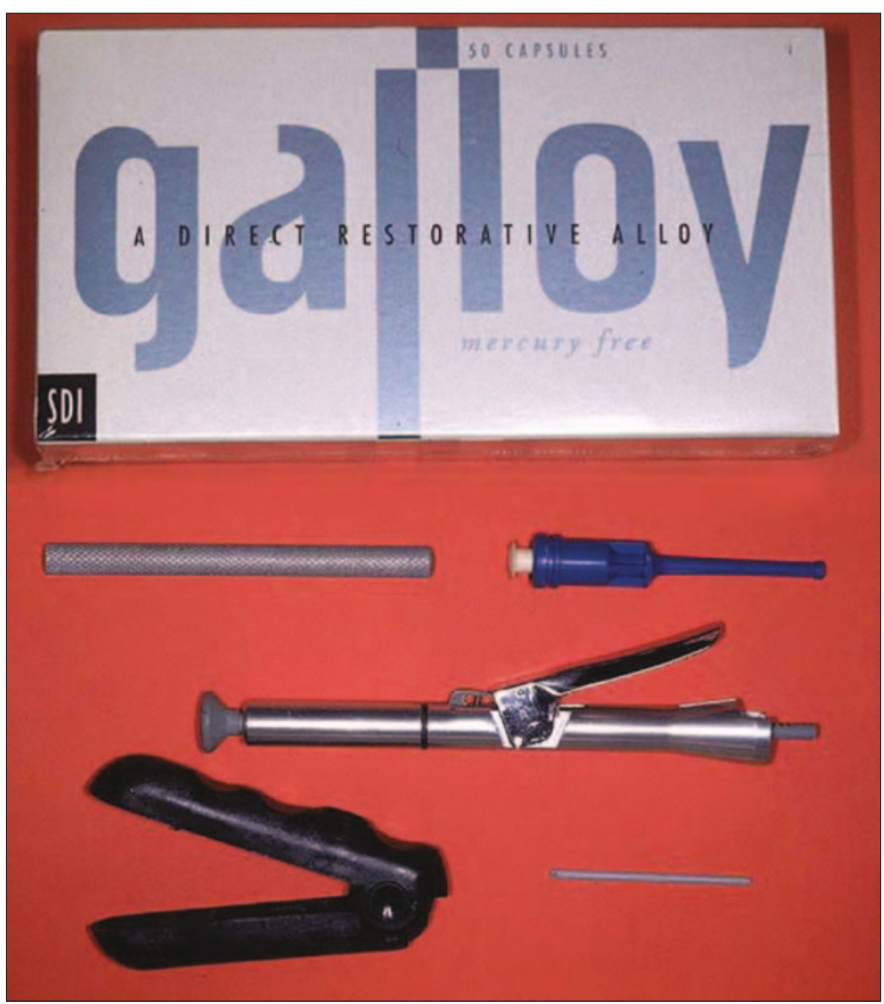

Fig. 1 The Galloy material and delivery system. Following trituration, the capsule is loaded into the side-action gun and the Galloy dispensed directly from the nozzle of the capsule.

reported no major problems associated with Galloy restorations other than tarnish and surface roughness. The authors emphasised the need to gain familiarity with the handling technique and the strict requirement to seal the restoration against fluid contamination in order to minimise corrosion product formation and excessive expansion. However, the 30 restorations placed in the clinical study by Osborne and Summit ${ }^{9,10}$ were very small class I restorations and the authors observed that the core of knowledge for the gallium alloy restoratives was limited and that studies on class II and extensive restorations should be conducted.

The present study reports the results of a 3-year longitudinal controlled clinical trial to compare the clinical performance of Galloy $^{\circledR}$ and Dispersalloy ${ }^{\mathrm{C}}$ (control) restorations in larger class I and class II cavities.

\section{MATERIALS AND METHODS}

Ethical committee approval was obtained for the study from Guy's, King's and St Thomas Ethics Committee. Patients were provided with an information sheet containing a detailed explanation of the study and were given an opportunity to reflect and time to ask questions on any aspect of the study. Patients signed a written consent form. The study population were 14 adult patients attending the Primary Dental Care Department. Patients were selected who had two or more cavities resulting from de novo carious lesions or failed existing restorations. The cavities were of moderate or large size with clinical indications for the use of amalgam as the restorative material. Moderate or large cavities were defined as cavities in which the bucco / palatal / lingual cavity width was equal to or exceeded approximately a third of the occlusal table or proximal surface. Indications for amalgam use included the following criteria:- remaining walls supported by dentine; cavity capable of being made mechanically retentive/resistive without undermining support and cavosurface angle capable of being finished to approximately $90^{\circ}-110^{\circ}$; cavity depth $2 \mathrm{~mm}$ without need for unnecessary removal of sound tooth structure. Restorations were placed in

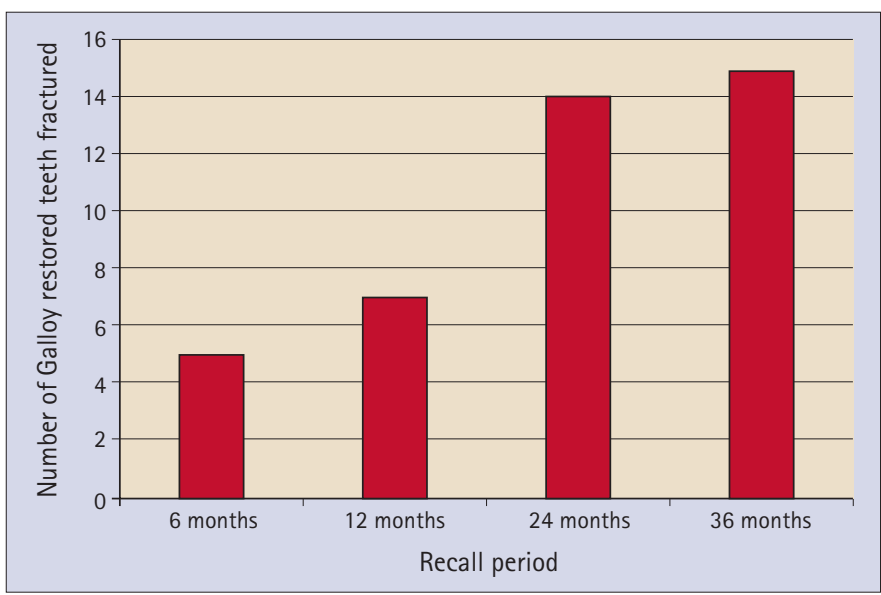

Fig. 2 Cumulative tooth cusp fracture associated with gallium alloy restorations.

alternating order starting with the control material Dispersalloy. A total of 50 restorations were placed in the 14 enrolled patients comprising 25 control restorations namely Dispersalloy (Dispersalloy, Dentsply, Milford, USA) and 25 test restorations with Galloy ${ }^{\circledR}$ (Galloy, Southern Dental Industries, Bayswater, Australia). The research commenced in 1998 and was supported by grants from Southern Dental Industries and Oral B. The Galloy restorations consisted of 2 class Is, 18 class IIs (MO or D0) and 3 pin-retained restorations. The Dispersalloy restorations comprised 3 class Is, 18 class IIs and 4 MODs. Rubber dam was employed for placement of all 50 restorations and all cavities were lined with a Vitrebond ${ }^{\circledR}$ (Vitrebond, 3M, St Paul, MN, USA) resin modified glass ionomer lining. Prior to Galloy placement, all cavities were coated with a film of low viscosity resin (triethylene glycol dimethacrylate, urethane dimethacrylate, EBDAMA and bis-GMA-containing resin), supplied by SDI. After carving, the Galloy, restorations were covered with an additional film of resin, strictly in accordance with the manufacturer's instructions. The occlusion was checked and if requiring modification, resin was reapplied to the Galloy surface. No resin was used in the cavities restored with Dispersalloy. All Galloy restorations were polished within one week of placement, as recommended by Osborne and Summitt. ${ }^{9}$ The restorations were then photographed and a silicone impression of the tooth containing the restoration recorded. Silicone impressions are undergoing profile examination. Patients were recalled at 6 months, 1 year and 2 and 3 years, the silicone impressions and photographs were repeated. Restorations were evaluated clinically at the recall appointments to assess tooth cusp fracture, percentage of surface tarnish and roughness, and fracture at the margins. Using visual inspection, tooth cusp fracture was scored as either complete loss of the cusp or as a hairline fracture. The latter was identified using transillumination. Percentage of surface tarnish, surface roughness and fracture of margins was estimated by a single operator at clinical examination following naked eye examination. The restorations were placed by a single operator. The Student's ttest was used for the statistical analysis, using a significance level of $\mathrm{p}<0.05$.

\section{RESULTS}

The handling characteristics of the Galloy restorative were slightly inferior to the Dispersalloy controls as the more fluid initial consistency of the Galloy rendered condensation more difficult. The fresh mix of Galloy was initially very plastic, allowing the alloy to stick to the condensing instrument. After initial practice, the SDI injection delivery system (see Figure 1) was easily mastered. The gallium alloy left a dark residue on latex gloves 


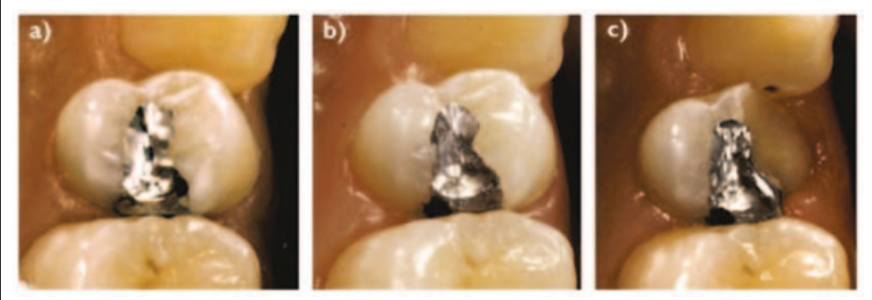

Fig. 3 Cusp fracture associated with a Galloy restoration a) Baseline b) 6 months c) 1 year.

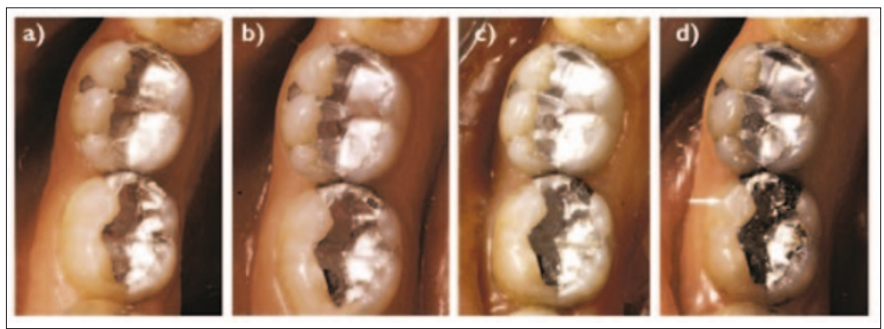

Fig. 4 Hairline cusp fracture (arrowed) associated with a Galloy restoration on the lower right second molar noted at 2-year recall, a) baseline,

b) 6 months, c) 1 year and d) 2 years. Dispersalloy control on the lower right first molar.

if handled. Cavity preparations were uneventful. No pulpal exposures or pre-existing cusp fractures were noted by the operator.

At 3 years the fate of 25 Galloy and 22 Dispersalloy restorations was known. Two patients with Dispersalloy restorations were lost from the study, which accounted for the missing data on three of the Dispersalloy restorations. The patients could not be contacted and data on the restorations was excluded from the study. The major differences in performance between the two materials were observed by the end of the second year as all 22 Dispersalloy restorations but only eight of the Galloy restorations were still in situ; three had previously been replaced due to persistent pain. By year three a further four Galloy restorations were lost spontaneously and a further three replaced due to serious pain, leaving only four remaining in situ. None of the Dispersalloy restoration had been removed over the three year assessment period.

Tooth fracture accounted for the majority of lost restoration in the Galloy group. Cumulative data on tooth cusp fracture associated with gallium alloy restoration can be seen in Figure 2. One third of the restorations were lost due to cusp fracture in the first six months after placement. Fifteen Galloy restorations (60\%) were associated with a tooth fracture and had either fallen out naturally

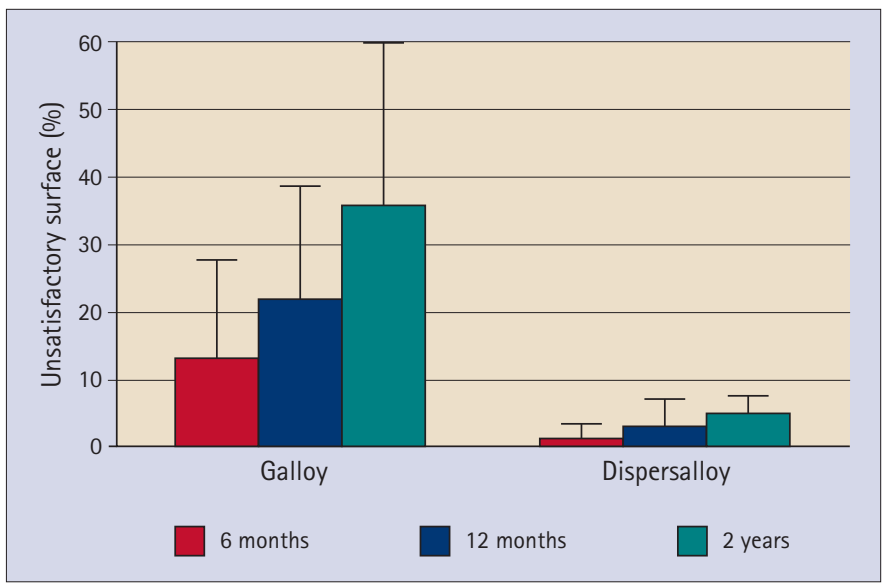

Fig. 5 Percentage of restoration surface unsatisfactory at 6 months, 1 year and 2 years after placement of Galloy and Dispersalloy restorations.

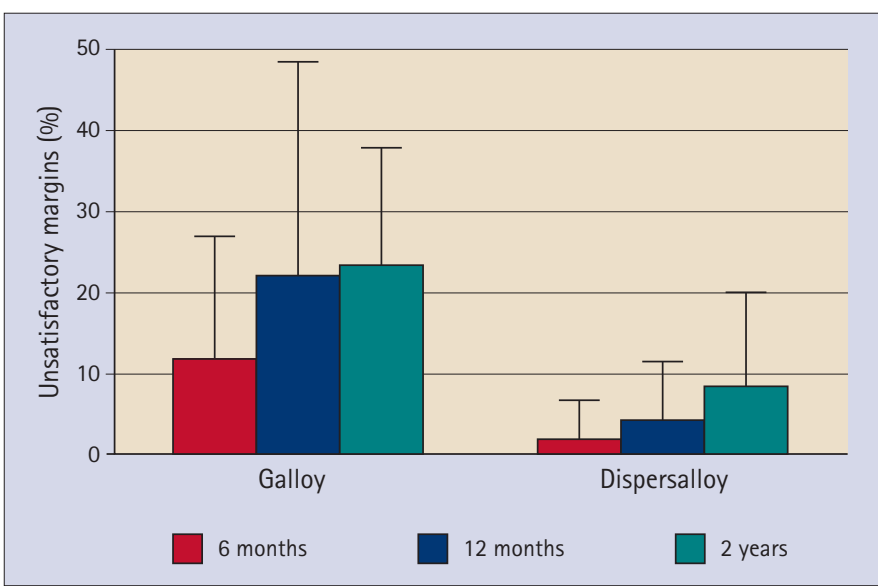

Fig. 6 Percentage of restoration margin unsatisfactory at 6 months, 1 year, and 2 years after placement of Galloy and Dispersalloy restorations.
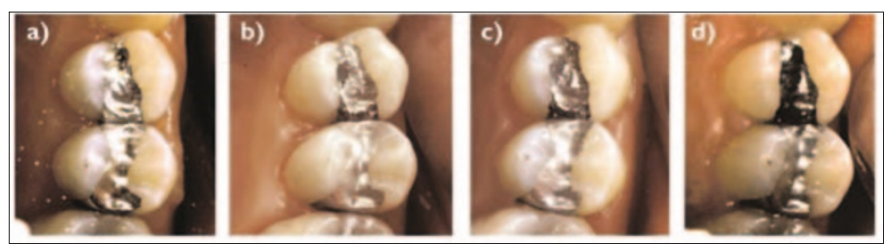

Fig. 7 Surface deterioration, corrosion and eventual cusp fracture of a DO Galloy restorations (upper first premolar) compared to the Dispersalloy control (upper left second premolar) at a) Baseline b) 6 months, c) 1 year d) 2 years and e) 3 years.

or been replaced by 3 years. Only 1 Dispersalloy restoration was associated with a tooth cusp fracture. Examples of cusp fractures associated with Galloy restorations are shown in Figures 3 and 4. Four teeth restored with Galloy and associated with cusp fracture subsequently required root canal treatment. No Dispersalloy restored teeth required endodontics.

The mean percentage of unsatisfactory margins and surfaces at four time intervals are show in Figures 5 and 6 . The mean percentages of unsatisfactory margins for the Galloy and Dispersalloy restorations at two years were $23.4(+/-14.3)$ and $8.7(+/-3.4)$, respectively. The difference between these means was significant $(\mathrm{P}<0.01)$. The mean percentages of unsatisfactory surfaces for the Galloy and Dispersalloy restorations at 2 years were $35.0(+/-24.0)$ and $4.5(+/-3.4)$, respectively. The difference between these means was significant $(\mathrm{P}<0.01)$. Examples of surface and margin deterioration of Galloy restorations and Dispersalloy controls are shown in Figure 7. Of the four Galloy restorations remaining in situ after three years, one moderate sized restoration required a marginal repair and the other three, which were relatively smaller restorations, were satisfactory.

\section{DISCUSSION}

Clinical reports on the use of gallium-based dental alloys are few in number and preliminary data available on Galloy at the commencement of this study in 1998 was limited, and was confined to class I restorations. ${ }^{9,10}$ This study was commissioned jointly by the manufacturer of Galloy and Oral-B with a view to introduction of the material into the UK. Galloy had met with statutory specifications including the USA Food and Drug administration following favourable clinical studies in the where Galloy was used in small cavities. ${ }^{9,10}$ At the 6-month recall the present authors were concerned with the high incidence of postoperative sensitivity associated with Galloy. ${ }^{11}$ At the one-year recall stage the authors had found sufficient problems with the clinical performance of the material that the manufacturer and Oral-B 
halted the formal study and withdrew further funding for the second year of the study. On the strength of our one-year study Oral-B declined to introduce Galloy to the UK market. With the agreement of the administration of the host institution, at each subsequent review the participants were informed of the results of the study to date and offered the option of replacement of Galloy restorations. The review process was continued for a further two years, by which time most of the Galloy restorations had been replaced due to occurrence of clinical problems with the restorations.

Manufacturer's data on the mechanical properties of Galloy indicated excessive expansion of the restorative material compared to conventional mercury amalgam if exposed to moisture in the first eight hours after placement. Hence the recommendation to use a resin liner and surface coating in conjunction with a rubber dam to reduce exposure to moisture during condensation and setting. ${ }^{9}$ Preliminary one-year interim data published by Osborne and Summitt ${ }^{9}$ using the moisture control method for direct placement found that all the Galloy class I restorations were in situ at the end of the year and no restoration fractures were observed. Although surface deterioration was noted: surfaces were generally rough, with 57\% exhibiting a tarnished surface but with little or no fracture at the margins. Whereas the data presented in this study on moderate to large class I and class II restorations demonstrated a remarkably different response at one year. Seven of the 25 Galloy restorations were lost as a result of tooth fracture, 25\% had demonstrated fracture at the margins and 20\% of the surfaces were tarnished and rough. Evidence of dimensional instability was observed early, as five Galloy restorations were associated with tooth cusp fracture by the first recall visit at six months. Thus, despite the great care taken in the placement of all restorations and, in particular, the avoidance of moisture contamination of the Galloy restorations, the incidence of tooth cusp fracture associated with Galloy was alarmingly high. This is in marked contrast to the findings of Osborne and Summitt ${ }^{9,10}$ where only small restorations were placed. The present authors suggest that these markedly different results are explained by the differences in cavity size and types in the two studies. The very small volume of restorative material and the integrity of the surrounding tooth structure in the Osborne and Summitt study ${ }^{9,10}$ contrasted with the larger restorations and thus weaker residual tooth structure in the present study. This interpretation is supported by results from a trial of class V Galloy restorations in primary teeth. ${ }^{12}$ In a controlled trial, Galloy restorations were placed in children between the ages of $5-7$ years and evaluated over a three month period. The Galloy restorations exhibited less marginal breakdown and improved marginal adaption compared to the control group treated with high copper amalgam restorations. Furthermore, no significant differences were observed between the test and the control groups with regard to lustre, texture, tooth discolouration or degree of microleakage. None of the Galloy restorations were lost as a result of fracture through the body of the restoration. ${ }^{12}$

However, problems arising from dimensional instability become apparent even in small restorations when observations are continued over a sufficiently long time span. Osborne and Summitt in reporting the results of their 3 year longitudinal study ${ }^{13}$ of Galloy in class I restorations found one fractured tooth at 21 months and a further two fractured restorations at three years in the 23 teeth that were assessed. The lack of control restorations in this survey may have complicated interpretation of the results, delaying recognition of the association between Galloy and tooth cusp fracture. Nevertheless the prognosis of Galloy in small restorations is still superior to that observed in larger multi-surface restorations as in this study where, after three years, only 4 of the 25 original Galloy restorations were still in situ. Possibly due to the smaller surface area to volume exposed to oral fluids and the improved visibility and ease of sealing the surface compared to restorations with an interproximal surface.

A similar high clinical failure rate was described in a one-year study comparing Galloy versus a high copper spherical alloy Tytin ${ }^{\circledR}$ in a mixture of class I and class II restorations. ${ }^{14}$ Forty-five per cent (28/65) of the Galloy restorations were removed or lost at one year due to fracture of restorations and/or teeth, sensitivity and loss of vitality compared to the failure of only one Tytin ${ }^{\circledR}$ restoration from an isthmus fracture. The failures occurred early, as by 9 weeks one tooth restored with Galloy ${ }^{\circledR}$ had fractured and by 15 weeks, another three teeth filled with Galloy were non-vital. ${ }^{14}$ The present authors have previously reported similar levels of sensitivity. ${ }^{11}$ In an interim report we found that Galloy restorations were associated with much greater severity of postoperative sensitivity than Dispersalloy restorations at six months (1.8 S.D. +/- 3.0 on a visual analogue scale compared to 0.2 S.D.+/$0.1 ; \mathrm{p}<0.01) .{ }^{11}$ Interestingly, the early breakdown of the restorations described by Neo et al. ${ }^{14}$ graphically illustrated the importance of the encapsulation procedures and the problems associated with moisture control. They used PAAMA 2, a dentine adhesive system (SDI, Bayswater, Australia) as the sealant agent. The sealant was lost from the surface of the restorations in less than 24 hours in contrast to the 2-4 weeks retention of the (triethylene glycol dimethacrylate, urethane dimethacrylate, EBDAMA and bis-GMAcontaining resin) sealant used in the 3-year longitudinal study of Osborne and Summitt ${ }^{14}$ and in this study. Furthermore the authors ${ }^{14}$ comment that subsequent research indicates that PMMA is permeable and does not bond to Galloy.

The corrosion properties and lack of dimensional stability of the Galloy restorations may have been responsible for many of clinical failures encountered in this study including sensitivity, loss of vitality and fractured tooth structure. Initial reports indicated that the in vitro setting expansion was documented as well within ISO limits and creep resistance and $1 \mathrm{hr}$ compressive strength have been found to be well above minimum requirements. ${ }^{7}$ The corrosion characteristics of Gallium Alloy GF have been found to be inferior to those of amalgam alloys. ${ }^{14}$ Corrosion could be accelerated by electrolytes in water. Venugopalan et $\mathrm{al}^{15}$ examined under in vitro test conditions the affect of water contamination on the dimensional stability and the corrosion properties of Galloy compared to a high copper spherical amalgam. Galloy exhibited contraction followed by expansion when contaminated with water during the condensation and setting process. Whereas post-setting exposure to water did not result in expansion of the gallium alloy when measured over 7 days. Although these evaluations were conducted on dry specimens over a short time period, the authors noted that the material exhibited a three fold greater susceptibility to corrosion than amalgam. They cautioned that delayed expansion could be deleterious to an already weakened tooth structure. ${ }^{15}$

Other authors have attempted to mimic exposure to $\mathrm{Cl}-$ electrolytes in saliva and blood during placement in the oral cavity and subsequent function of the restoration. Osborne ${ }^{16}$ employing a photoelastic resin to assess three-dimensional expansion confirmed that Galloy contaminated with water demonstrated the same expansion as uncontaminated Galloy. The experiments were then repeated using Ringer's solution or cell culture medium to simulate saliva and blood products. However, when Galloy was exposed either for 5 minutes to mimic moisture exposure during condensation or alternatively for 3 days to simulate exposure during the setting reaction, excessive expansion of Galloy compared to Dispersalloy was observed. By six weeks the Galloy restorations exposed to Ringers solution had been extruded from the mould. The restorations were extruded from the surface of the blocks by $2.1 \mathrm{~mm}$ in 3 months which is several orders of magnitude greater than the $22.56 \mu \mathrm{m}$ expansion recorded by Venugopalan et al. ${ }^{15}$ after water contamination during condensation of the restorative 
material. In addition, in test blocks where there was minimal extrusion of the alloy restorations, very high stress measurements were recorded from within the block. Such a build up of stress could account for fracture of teeth or restoration, and sensitivity observed in clinical studies.

The affect of post-placement delayed expansion could result in the generation of cracks throughout the structure. It is postulated that the cracks would precipitate a crevice corrosion effect, which could exacerbate the corrosive breakdown of the alloy and enhance dimensional instability. ${ }^{14}$ The corrodant electrolytes from the mouth diffuse into the restoration leading to internal corrosion which in turn provides corrosion products that feed the surface corrosion. The corrosion products have a higher molecular volume than the original constituents hence the expansion seen as the material corrodes. As the internal corrosion products increase the Gallium will creep and extrude along the norm to the surface, or if inhibited from extruding by the restraints of the cavity preparation will expand with the potential to crack the tooth or cause post-operative sensitivity. ${ }^{17}$ The internal corrosion is considered to be responsible for the loss of strength and disintegration at the margins observed in this and other studies. ${ }^{13,14}$ In addition the expansion could break the restoration seal and lead to microleakage, resulting in secondary caries with the potential for sensitivity and pulpal involvement. ${ }^{17}$

In conventional amalgams the controlled formation of adherent corrosion products is considered beneficial as they improve marginal adaption. But the uncontrolled and continuing growth of internal corrosion products in Gallium materials can lead to dimensional change in the order of $21.5 \%$, which far exceeds the limit of $0.02 \%$ set by the American Dental Association for dimensional change. ${ }^{16}$

In conclusion the excessive internal and external corrosion of Galloy is inherent in its heterogeneous microstructure and multielement composition. All the constituent components with the exception of Ag and Pt are highly electroactive and form corrosion products in the aqueous electrolytes that naturally bathe the teeth, with the result that there is a lack of tolerance to moisture contamination. The strenuous attempts to encapsulate the restorations from dentine tubular secretions with cavity liners and surface protection with sealants were insufficient to override intrinsic physical and chemical properties of the material. The observed catastrophic failure rate resulting in cusp fracture in association with
$60 \%$ of the restorations makes Galloy an unsuitable as a restorative material in its current formulation. Deterioration of the margins and surfaces of the Galloy restorations was much greater than that of the controls and overall the clinical performance of Galloy restorations was grossly inferior to that of the Dispersalloy controls. The authors cannot recommend the use of Galloy in moderate to large class I and class II cavities. The manufacturer's decision to withdraw Galloy from the UK market based on the one year's results from this study prevented potential damage to many thousands of patient's teeth in the UK. Galloy is still employed in some parts of the world and we feel that the major problems identified in this clinical study should be widely disseminated.

1. Puttkammer A. Mercury-free amalgam? Zahnaerztl Rundsch 1928; 35: 1450-1454. 2. Smith D L, Caul H J. Alloys of gallium with powdered metals as possible replacements for dental amalgam. JAm Dent Assoc 1956; 53: 315-324.

3. Smith D L, Caul H J Sweeney W T. Some physical properties of gallium-copper-tin alloys. JAm Dent Assoc 1956; 53: 677-685.

4. Waterstrat R M. Evaluation of gallium-palladium-tin alloy for restorative dentistry. JAm Dent Assoc 1969; 78: 536-541.

5. Dunne S M, Wilson N, Gainsford I D. Current material and techniques for direct placement restorations in posterior teeth. Part 1: Silver amalgam. Int Dent J 1997 47: 123-136.

6. Wilson N, Dunne S M, Gainsford I D. Current material and techniques for direct placement restorations in posterior teeth. Part 2: Composites. Int Dent J 1997; 47: 185-193

7. Hero H, Okabe T. Gallium alloys as dental restorative materials: A research review. Cells Mater 1994; 4: 409-418.

8. Mash L K Miller B H, Nakajima $H_{1}$ Collard S M, Guo | Y, Okabe T. Handling characteristics of gallium alloy for dental restorations. J Dent 1993; 21:350-354.

9. Osborne J W, Summitt J B. Mechanical properties and clinical performance of a gallium restorative material. Oper Dent 1995; 20: 241-245.

10. Osborne J W, Summitt J B. 2-year clinical evaluation of a gallium restorative alloy. Am J Dent 1996; 9: 191-194.

11. Dunne S M, Abraham R. Dental post-operative sensitivity associated with a galliumbased restorative material. Br Dent J 2000; 189: 310-313.

12. Munshi A K, Hegde A M, Bhaskar S. Gallium alloy versus high copper amalgam: a comparative evaluation of corrosion resistance and microleakage in the primary teeth. J Clin Pediatr Dent 2000; 24: 315-319.

13. Osborne J W, Summitt J B. Direct-placement gallium restorative alloy: A 3-year clinical evaluation. Quintess Int 1999; 30: 49-53.

13. Neo J Chew C L, Osborne J W, Mahler D B. Clinical evaluation and microstructura analysis of a direct placement gallium restorative alloy. J Dent 2000; 28: 123-129.

14. Oshida $Y$, Moore B K. Anodic polarization behaviour and microstructure of gallium-based alloy. Dent Mat 1993; 9: 234-241.

15. Venugopalan $R$, Broome J C, Lucas L C. The effect of water contamination on dimensiona change and corrosion properties of a gallium alloy. Dent Mat 1998; 14: 173-178.

16. Osborne J W. Photoelastic assessment of the expansion of direct-placement gallium restorative alloys. Quintess Int 1999; 30: 185-191.

17. Sarkar N K, Moiseyeva R, Berzins D W, Osborne J W. Long-term corrosion of a Gacontaining restorative material. Dent Mat 2000; 16:97-102. 